ARTICLE

\title{
Depth-dependent valence stratification driven by oxygen redox in lithium-rich layered oxide
}

Jin Zhang 1,2,3,8, Qinchao Wang ${ }^{4,8}$, Shaofeng Li ${ }^{2}$, Zhisen Jiang ${ }^{2}$, Sha Tan ${ }^{4}{ }^{4}$, Xuelong Wang ${ }^{4}$, Kai Zhang ${ }^{1}$, Qingxi Yuan (10 ${ }^{1 \times}$, Sang-Jun Lee (10 ${ }^{2}$, Charles J. Titus (1) ${ }^{5}$, Kent D. Irwin (10 5 , Dennis Nordlund (1) ${ }^{2}$, Jun-Sik Lee (i] ${ }^{2}$, Piero Pianetta ${ }^{2}$, Xiqian Yu (iD ${ }^{6}$, Xianghui Xiao (iD ${ }^{7}$, Xiao-Qing Yang (iD ${ }^{4}$, Enyuan Hu (i) ${ }^{4 凶} \&$ Yijin Liu (1) 2 四

Lithium-rich nickel-manganese-cobalt (LirNMC) layered material is a promising cathode for lithium-ion batteries thanks to its large energy density enabled by coexisting cation and anion redox activities. It however suffers from a voltage decay upon cycling, urging for an in-depth understanding of the particle-level structure and chemical complexity. In this work, we investigate the $\mathrm{Li}_{1.2} \mathrm{Ni}_{0.13} \mathrm{Mn}_{0.54} \mathrm{Co}_{0.13} \mathrm{O}_{2}$ particles morphologically, compositionally, and chemically in three-dimensions. While the composition is generally uniform throughout the particle, the charging induces a strong depth dependency in transition metal valence. Such a valence stratification phenomenon is attributed to the nature of oxygen redox which is very likely mostly associated with Mn. The depth-dependent chemistry could be modulated by the particles' core-multi-shell morphology, suggesting a structural-chemical interplay. These findings highlight the possibility of introducing a chemical gradient to address the oxygenloss-induced voltage fade in LirNMC layered materials.

\footnotetext{
${ }^{1}$ Beijing Synchrotron Radiation Facility, Institute of High Energy Physics, Chinese Academy of Science, 100049 Beijing, China. ${ }^{2}$ Stanford Synchrotron Radiation Lightsource, SLAC National Accelerator Laboratory, Menlo Park, CA 94025, USA. ${ }^{3}$ University of Chinese Academy of Sciences, 100049 Beijing, China. ${ }^{4}$ Chemistry Division, Brookhaven National Laboratory, Upton, NY 11973, USA. ${ }^{5}$ Department of Physics, Stanford University, Stanford, CA 94305, USA. ${ }^{6}$ Beijing Advanced Innovation Center for Materials Genome Engineering, Key Laboratory for Renewable Energy, Beijing Key Laboratory for New Energy Materials and Devices, Institute of Physics, Chinese Academy of Sciences, 100190 Beijing, China. ${ }^{7}$ National Synchrotron Light Source II, Brookhaven National Laboratory, Upton, NY 11973, USA. ${ }^{8}$ These authors contributed equally: Jin Zhang, Qinchao Wang. ${ }^{凶}$ email: yuanqx@ihep.ac.cn; enhu@bnl.gov; liuyijin@slac.stanford.edu
} 
ithium-ion battery (LIB) is crucial for a world based on clean energy. Its importance has recently been recognized by the awarding of 2019 Nobel prize in chemistry to scientists working in this field ${ }^{1}$. Despite its great success, there is always strong motivation to further increase the energy density of LIB, especially when it comes to the application in large systems such as electric vehicles. To reach this goal, an effective approach is to increase the energy density of the cathode, a major component of the LIB. Conventional cathodes are solely based on transition metal (TM) cation redox, limiting the maximum capacity and energy density that can be possibly achieved. In this context, lithium-rich nickel-manganese-cobalt (LirNMC) layered material stands out as a high energy cathode by utilizing both cation and anion redox ${ }^{2-7}$. Typically, LirNMC can deliver capacity up to $280 \mathrm{mAh} \mathrm{g}^{-1}$. Unfortunately, this material has not been put into practical application because its voltage keeps decreasing during cycling (commonly referred to as "voltage fade" in the battery community). Many research efforts have been devoted to understanding the origin of such voltage fade ${ }^{8-11}$, mostly at the atomic or the electrode level. To bridge the gap in the length scale, it is highly desirable to understand the material at the particle level, which is termed the mesoscale.

Material properties at the mesoscale are important for material design. For example, mesoscale structural and chemical defects were found to play a very significant role in affecting the overall electrochemical performance of the battery ${ }^{12,13}$. Depthdependent redox heterogeneity within the cathode particles has also been reported to affect the charging behavior ${ }^{14}$ and the thermal stability ${ }^{15-17}$. It can be expected that compositional, chemical, and morphological understanding of LirNMC material at the mesoscale will provide insight into the mechanism of voltage fade issue and suggest possible measures to address this challenge.

In this work, we employ nano-resolution spectro-tomography to study a typical LirNMC material- $\mathrm{Li}_{1.2} \mathrm{Ni}_{0.13} \mathrm{Mn}_{0.54} \mathrm{Co}_{0.13} \mathrm{O}_{2}$, spatially resolving the morphological, compositional, and chemical features in three-dimensions (3D) within the LirNMC particles $^{18-20}$. We observed a multi-layer morphology, which is very likely correlated with the depth-dependent chemistry. We further reveal the chemical and spatial dependence of oxygen redox behavior in LirNMC material, providing an in-depth understanding of the voltage fade issue. These findings suggest that a depth-dependent compositional engineering strategy ${ }^{21}$ could be a viable path for solving the voltage fade problem in the LirNMC material.

\section{Results}

The composite battery cathode electrode is made of a large amount of several micron-sized active particles that are imbedded in a porous matrix of conductive carbon and binder domain (CBD). The active cathode particles are, ultimately, the energy reservoirs in the battery and their functionality relies on the CBD to provide mechanical support, electrical conductivity, and ion diffusion pathways. The synchrotron-based x-ray tomography technique has been demonstrated as a powerful tool for noninvasively reconstructing the $3 \mathrm{D}$ morphology of the cathode from the cell ${ }^{22}$ and the electrode ${ }^{23}$ scales down to the particle level ${ }^{24-}$ 29. As shown in Fig. 1a, the structure and morphology of the pristine material are characterized by x-ray diffraction (XRD) and scanning electron microscopy (SEM), respectively. Detailed results of the Rietveld refinement of LirNMC material are shown in Supplementary Table 1. XRD pattern indicates there is a honeycomb superstructure in the Li/TM layer as evidenced by the superlattice peaks on the right shoulder of the strongest XRD peak (lowering the space group symmetry from $\mathrm{R} \overline{3} \mathrm{~m}$ to $\mathrm{C} 2 / \mathrm{m}$ ).
Recent studies by House and Bruce et al suggest that the honeycomb superstructure is closely related to the formation of $\mathrm{O}_{2}$ and oxygen vacancies ${ }^{30}$. The charge-discharge profile and the electrochemical cycling data are shown in Fig. 1b. The first cycle charge profile features a long plateau that is typical of the lithiumrich layered material and contributes to around two thirds of the whole charge capacity. As illustrated in Fig. 1c, we utilized the xray tomography to reconstruct the structure of a small piece of the LirNMC electrode. The studied electrode consists of a thin layer of active material and CBD (nearly a monolayer of LirNMC particles) on top of the aluminum current collector. High mass loading and close particle packing in a thick electrode $(40-60 \mu \mathrm{m}$ thick) is a common approach for improving energy density, which, however, can lead to significant particle-to-particle variation $^{23,31,32}$. In our study, we choose to focus on the particle level because our scientific focus is the interplay between the particle's morphology and the particle's internal redox stratification. Therefore, a low mass loading is purposely chosen to minimize the cell polarization effect. More details about the electrode fabrication procedure can be found in the method section. To zoom in to the sub-particle level microstructures, we utilized x-ray nano-tomography to reconstruct the morphology of a few randomly selected LirNMC cathodes particles (2D projection images of several randomly selected particles are shown in Supplementary Fig. 1). As shown in Fig. 1c (panel I, II, and III), these LirNMC particles have multi-layer morphologies, distinguishing LirNMC from other cathode materials ${ }^{33,34}$. Although such morphology has been hinted previously in two-dimensional images ${ }^{35}$, our three-dimensional tomographic data offers unambiguous visualization and quantification, which we will demonstrate in detail later in this work. The multi-layer morphology may be due to intrinsic complexities of LirNMC. For example, LirNMC has multiple cations that differ greatly in size and reactivity. LirNMC also has structural complexity and the nature of its structure (whether a solid solution or a composite) has been debated for a long time $\mathrm{e}^{36-38}$. The multi-layer configuration may have a profound impact on the redox chemistry within the particles as it adds more complexities to the lithium-ion diffusion and transition metal redox behavior within the particle.

To gain the depth profile of transition metal valence, we turned to the spectro-tomography technique ${ }^{39-42}$ which could spatially resolve the local valence state of the element of interest in $3 \mathrm{D}$. Such capability is achieved by conducting tomographic scans at a number of different energy levels across the absorption edge of the targeted element of interest. This technique is first applied to Mn, the most abundant transition metal in LirNMC. Two particles at the pristine and the charged (to $4.8 \mathrm{~V}$ in the initial cycle, at the fully charged state) states are scanned. As shown in Fig. 2a, d, the particle morphology is fairly similar and their Mn K-edge spectroscopic fingerprints over different regions (see labeling in the virtual slices through the center of the particle) are shown in Fig. $2 \mathrm{~b}$ and e, respectively. The pristine particle appears to be relatively homogeneous in the Mn's valence state as indicated by the depth-dependent Mn XANES plots in Fig. $2 \mathrm{~b}$ and the $3 \mathrm{D}$ rendering of the $\mathrm{Mn}$ K-edge energy distribution in Fig. 2c. On the other hand, the charged particle clearly shows a depth-dependent Mn redox variation (see Fig. 2e, $f, g)$. The core and the surface layer of the charged LirNMC particle exhibits high $\mathrm{Mn}$ valence state at $4^{+}$, while the $\mathrm{Mn}$ cations in the transition layers appear to be relatively reduced. These visual assessments of the pristine and the charged particles are further confirmed by the depth profile of Mn's K-edge energy shown in Fig. 2h. While the relative homogeneity of the Mn valence state distribution in the pristine particle is anticipated ${ }^{43}$, the observed depth-dependent $\mathrm{Mn}$ valence in the charged LirNMC particle is somewhat a surprise, specifically because of 
a

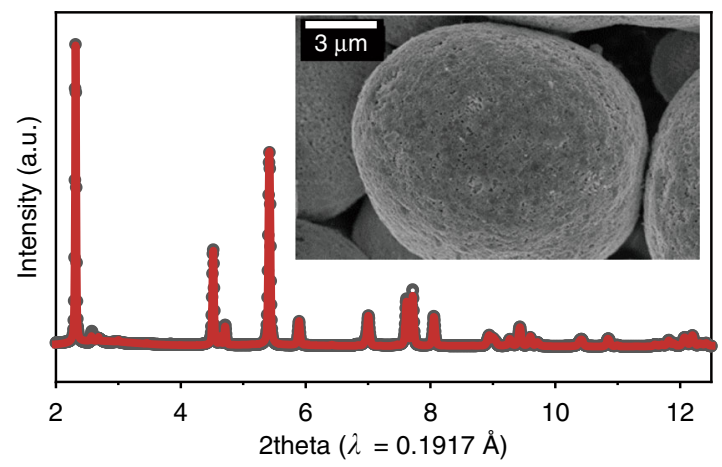

b

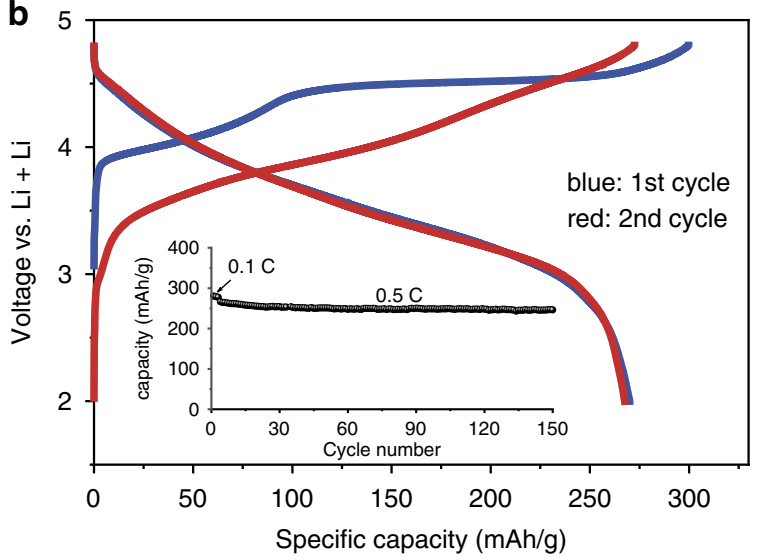

C

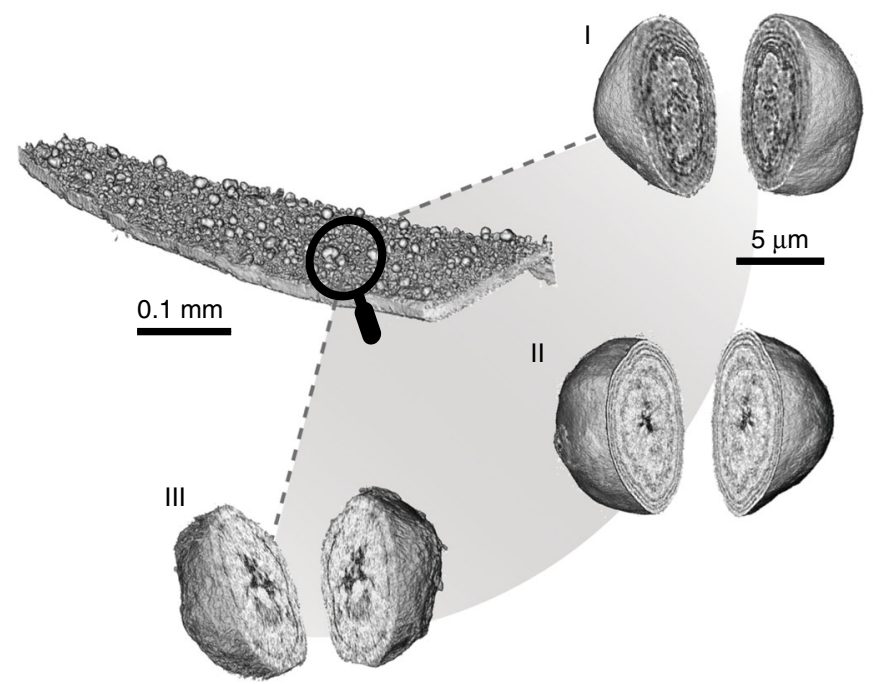

Fig. 1 Electrochemical performance and morphology of LirNMC. a Structural characterization of LirNMC cathode material by XRD and Rietveld refinement. The inset graph shows the SEM image of LirNMC particles. $\mathbf{b}$ Charge-discharge profile and electrochemical cycling data of LirNMC I |Li metal half-cell. c Three-dimensional rendering of the composite electrode and magnified views of a few arbitrarily selected LirNMC cathodes particles (panels I, II, and III). The mesoscale core/multi-shell separation is clear visible in all the particles.

its non-monotonicity. This observation motivates a more systematic study of all the transition metal cations ( $\mathrm{Mn}, \mathrm{Co}$, and $\mathrm{Ni}$ ) in the LirNMC particle in a correlative manner.

With the above-mentioned motivation, we carry out spectrotomographic scans of a charged LirNMC particle over the absorption K-edges of the $\mathrm{Mn}, \mathrm{Co}$, and $\mathrm{Ni}$, respectively, and correlatively. As shown in Fig. 3a, a depth-dependent redox heterogeneity can be observed in all the transition metal cations. To evaluate whether or not the compositional heterogeneity plays a role here, we first utilize the edge jump measurements on the Kedges of $\mathrm{Mn}, \mathrm{Co}$, and $\mathrm{Ni}$ (see Fig. 3b) to quantify the local elemental concentrations of these three metal elements ${ }^{44}$. The 3D elemental distributions are further quantified by evaluating their respective depth dependency (Fig. 3c), which suggests that the studied particle is relatively compositionally homogeneous throughout the particle volume. Correlating each pixel with edge position not only enables direct evaluation of 2D/3D images, but also provides a relative probability distribution as shown in Fig. 3d. The number of pixels featuring certain energy is divided by the number of total pixels to obtain the relative probability at that energy. Based on such probability distribution, we can further quantify the degrees of redox heterogeneity using the histogram plot of the 3D matrixes of edge energy. As shown in Supplementary Figs. 2-5, there are close relationship between edge position of the spectra and the valence of the transition metal. Therefore, the shape of the peak represents the relative probability distribution of the edge energy and the width of the peak effectively measures the degree of chemical nonuniformity. As shown in Fig. 3d, the Co's valence state shows larger full width at half maximum (FWHM) in its histogram plot than that of the
$\mathrm{Mn}$ and Ni. A quantitative evaluation of transition metal valence as a function of depth is shown in Fig. 3e. The valence of $\mathrm{Ni}$ is considerably low in the outermost layer and then gradually increases towards the core of the particle. The valences of $\mathrm{Mn}$ and Co are high in the outermost layer and then decrease going through the intermediate layer before increasing to high valence in the core area. In addition, in situ x-ray absorption spectroscopy (XAS) experiment at $\mathrm{Ni}, \mathrm{Mn}$, and Co K-edges was carried out (Supplementary Fig. 6) and the transition metal (mostly Ni) XAS spectra show obvious edge shift before the $4.5 \mathrm{~V}$ plateau and show no shift after the plateau begins. This suggests that as the oxidation of transition metal (mostly $\mathrm{Ni}$ ) takes place way before the end of charge (transition metal oxidation contributes only around one third of the total capacity, followed by oxygen oxidation which contributes to two thirds), the low valence of transition metal is unlikely caused by insufficient oxidation, but by the oxygen redox around the transition metal cations. To confirm that the transition metal's low valence is associated with oxygen redox, we further conduct spectro-tomography and compare the depth dependency of the redox profile (single pixel resolution) on particles at different states of charge $(4.3$ and $4.8 \mathrm{~V}$ in the first cycle) and after long-term battery operation (at $4.8 \mathrm{~V}$ after 50 cycles) shown in Fig. $4 \mathrm{a}$ for $\mathrm{Ni}, \mathrm{Mn}$, and Co, respectively. During the $1^{\text {st }}$ cycle charging (going from 4.3 to $4.8 \mathrm{~V}$ ), the valence profiles of $\mathrm{Ni}$ and $\mathrm{Mn}$ change significantly and in very different ways while the profile of Co is relatively stable. At intermediate depths (excluding the surface vicinity as well as the center), the oxidation states increase for $\mathrm{Ni}$ but decrease for $\mathrm{Mn}$. The $\mathrm{Ni}$ valence increase may be caused by incomplete Ni oxidation at 4.3 $\mathrm{V}$ and further charging leads to its valence increase. For Mn, the 
a
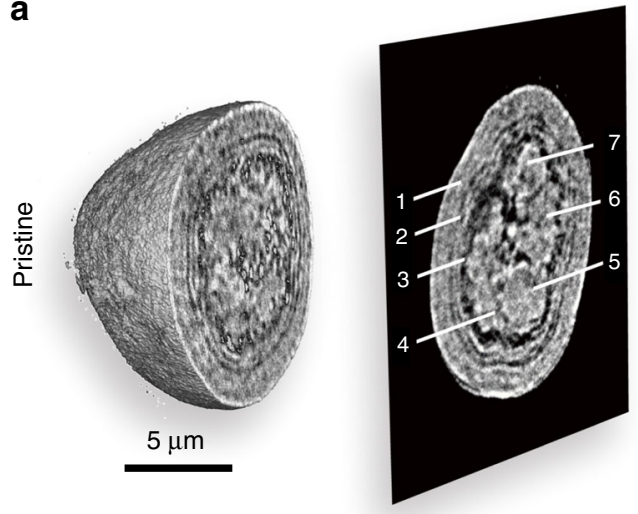

d
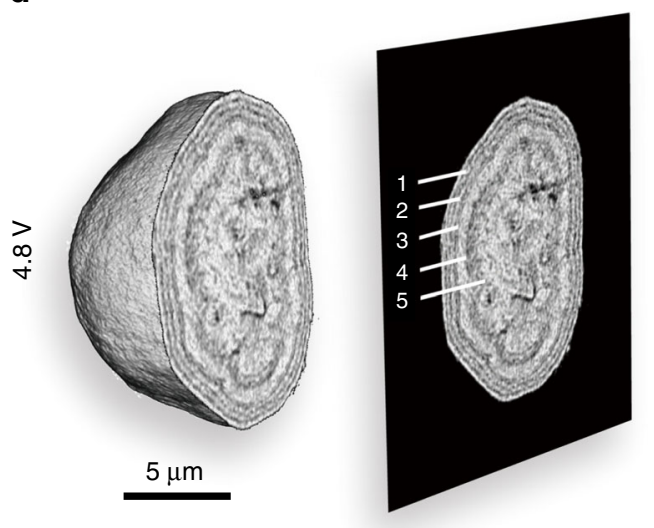

b

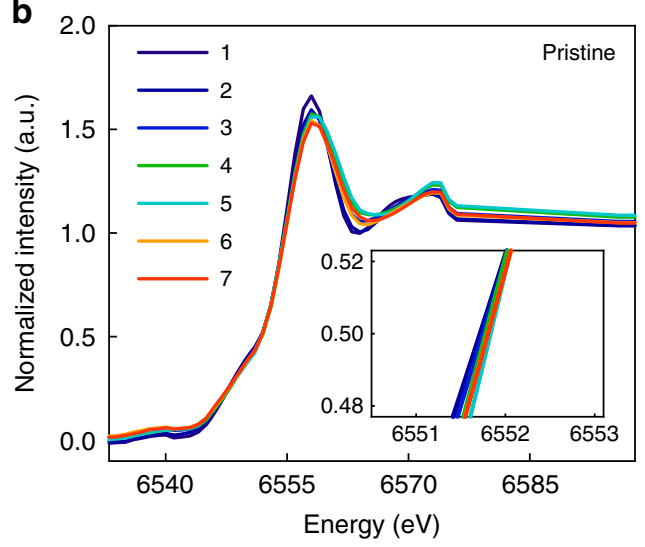

e

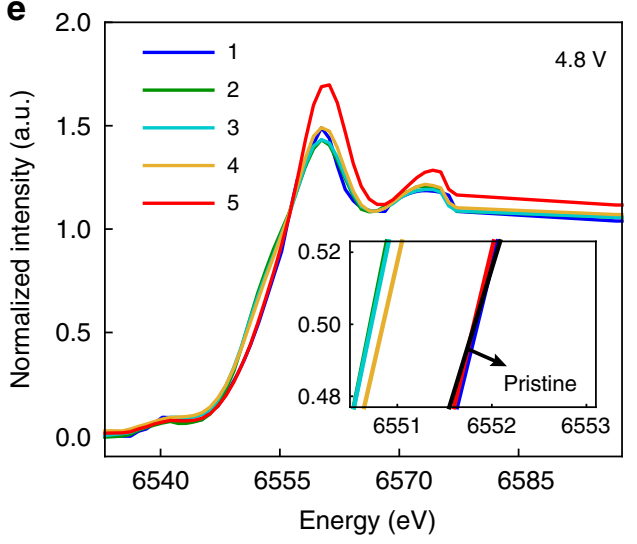

C

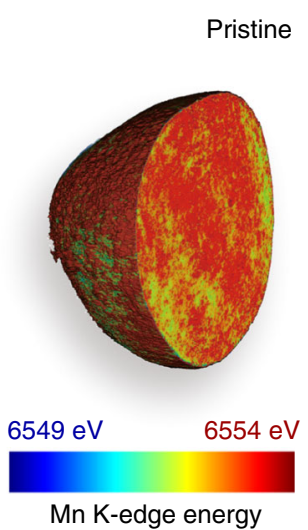

f

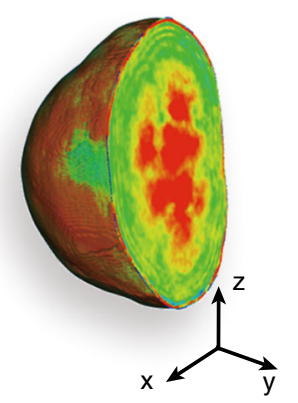

g
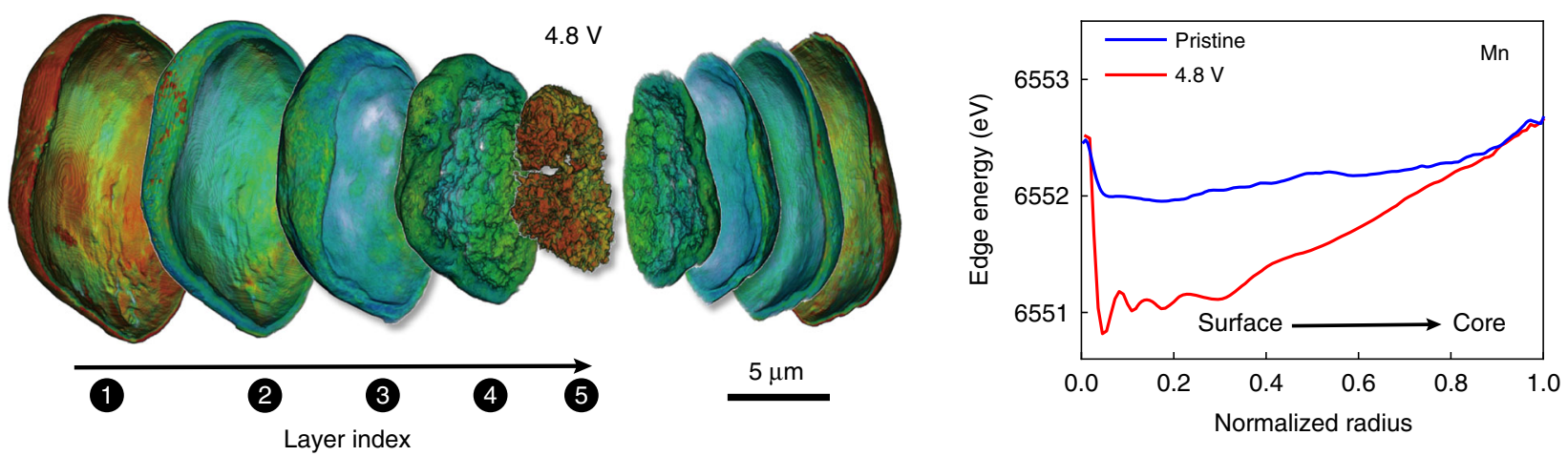

Fig. 2 Spectro-microscopic investigation of the LirNMC particles. Panels (a) to (c) are on a pristine particle and panels (d) to ( $\mathbf{g}$ ) are on a charged particle (at $4.8 \mathrm{~V}$ in the first charge). Panels (a) and (d) are the 3D visualizations of the pristine and charged particles, with the labeling of different regions of interest on the respective central $x z$-slices. Panels (b) and (e) are the Mn K-edge $\mathrm{x}$-ray absorption spectroscopic fingerprints over the regions of interest shown in panels (a) and (c), respectively. Panel (c) and (f) are the 3D renderings of the Mn's valence state distribution in the pristine and the charged particles. Panel ( $\mathbf{g})$ is a detailed layer-by-layer rendering of the Mn's valence state in the charged particle. Panel (h) is the comparison of the depth profiles of the particles in panels (c) and (f).

valence decreases going from 4.3 to $4.8 \mathrm{~V}$. This may be because of the oxygen redox and the largest part of it could be associated with Mn. Detailed reasons will be explained later when discussing the valence profile change during cycling. The outcome of oxygen redox may be in the form of $\mathrm{O}^{-45,46}$, oxygen vacancy ${ }^{9}$, or $\mathrm{O}_{2}$ in the lattice ${ }^{30}$. They all can lead to a decrease in the valence of TM, or very likely Mn. For Co, it is well known that Co can form a very strong covalent bond with oxygen and this may contribute to the relatively unchanged valence profile of Co during charging. In the surface vicinity, or in the passivation layer, $\mathrm{Ni}$ and $\mathrm{Mn}$ can also behave differently. For example, $\mathrm{NiMn}_{2} \mathrm{O}_{4}$ is a commonly seen spinel phase arising from loss of lithium and oxygen in the layered material ${ }^{9}$. In $\mathrm{NiMn}_{2} \mathrm{O}_{4}, \mathrm{Ni}$ is in a low valence state (divalent) while $\mathrm{Mn}$ is in high valence state (between trivalent and tetravalent and can be higher if it is partially substituted by $\mathrm{Li}$ ). In other words, the reconstructed surface may have low valence $\mathrm{Ni}$ but high valence Mn. Upon cycling, the valence profile evolves in different ways for $\mathrm{Ni}, \mathrm{Mn}$, and $\mathrm{Co}$. The tetravalent $\mathrm{Ni}$ has been well known to have high oxygen partial pressure and oxygen around $\mathrm{Ni}$ can be easily released. This can lead to $\mathrm{Ni}$ valence 
a
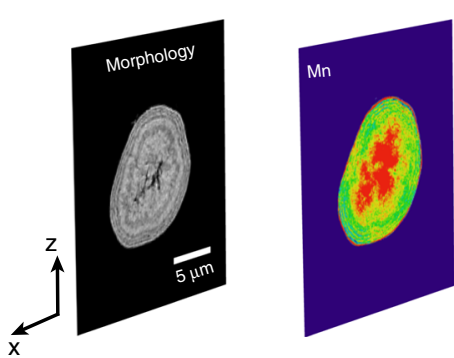

b

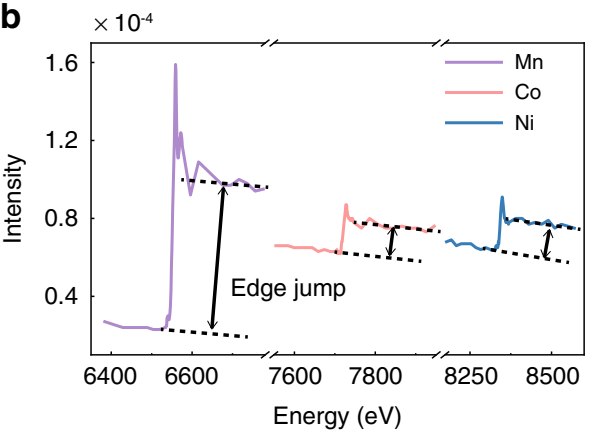

d

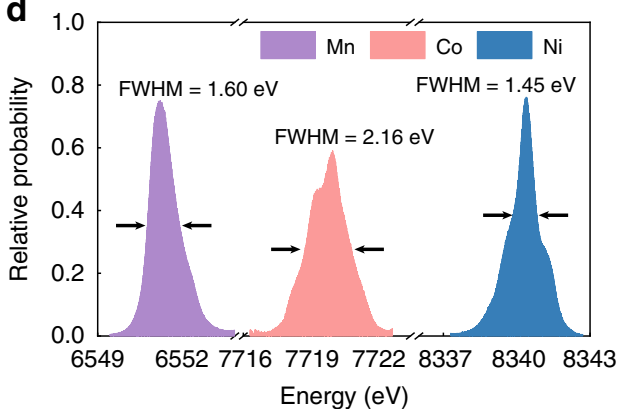

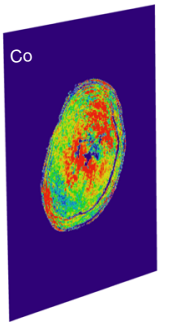
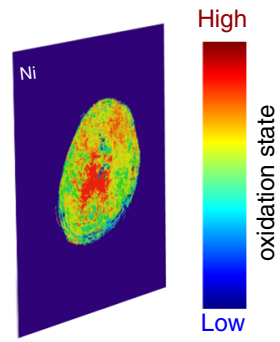

C

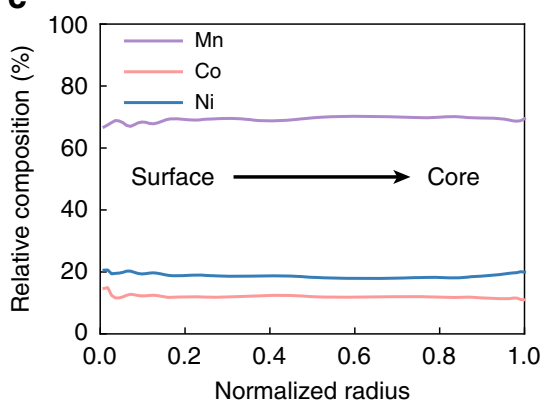

e

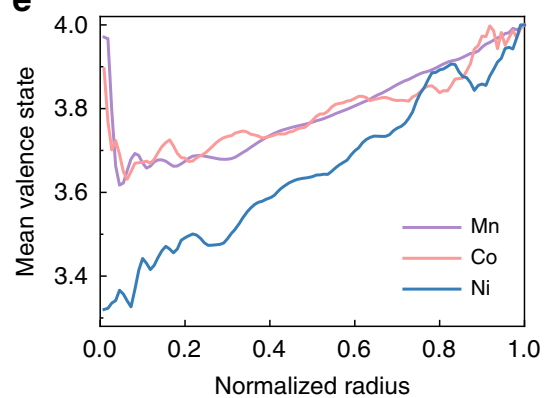

Fig. 3 The spatial variation of the composition and the valence states of $\mathbf{M n}, \mathrm{Co}$, and $\mathbf{N i}$ in a charged (to $\mathbf{4 . 8} \mathrm{V}$ ) LirNMC particle. Panel (a) shows the valence states of $\mathrm{Mn}, \mathrm{Co}$, and Ni over the central xz slice of a charged LirNMC particle. Panel (b) illustrates the x-ray absorption spectroscopic fingerprints over a typical voxel in the reconstructed spectro-tomographic data. The edge jump is proportional to the relative elemental concentration and is utilized to quantify the elemental distribution in the particle volume. Panel (c) is the depth-dependent elemental concentration over the studied particle, suggesting the compositional homogeneity throughout the particle. Panel (d) is the relative probability distributions of the edge energy over the K-edges of Mn, Co,

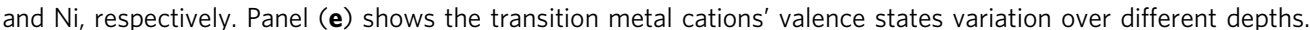

decrease and may be related to the voltage fade issue. Our previous work suggested that continuous oxygen loss from the lattice is one of the main reasons for voltage fade in lithium-rich layered material. Similarly, the decrease of Co valence during cycling may also be linked with the voltage fade issue. For $\mathrm{Mn}$, the valence profile shows relatively little change upon cycling. Interestingly, Gent et al showed that oxygen redox can be fairly reversible during cycling as indicated by the clearly visible oxygen oxidation RIXS feature in the long cycled sample ${ }^{46}$. These two observations may indicate that the largest part of reversible oxygen redox is coupled to Mn cations. Such argument is also supported by theoretical calculations showing that oxygen redox mainly takes place on those oxygen anions in the $\mathrm{Li}-\mathrm{O}-\mathrm{Li}$ moiety which has $\mathrm{Li}$ in the $\mathrm{Li} / \mathrm{TM}$ layer ${ }^{47}$. The monovalent $\mathrm{Li}$ is most likely surrounded by high valent TM such as Mn (maybe also Co) because this helps to release the strain ${ }^{48}$. In other words, the active oxygen anions are most likely around the Mn cations.

In addition to the evolution of the redox heterogeneity, we conducted additional synchrotron nano-tomography on particles after 50 electrochemical cycles at a rate of $0.5 \mathrm{C}$ and observed that clear cracks are populated in the particles after long-term cycling. It is useful to point out that the cracks are not confined in the particle core regions. Some of the cracks propagate through multiple shell layers, effectively open up the interior of the particle to the liquid electrolyte, forming fresh active solid-to-liquid interface that could facilitate the lithium exchange between the particle and the external environment (Supplementary Figure 7). The chemomechanical interplay in battery cathode particles is an active research field. Follow-up efforts for studying the particle morphological control as a viable method to mitigate the particle disintegration effect is highly desirable.

Valence heterogeneity is not a surprise as it has been observed in several cathode materials $22,27,33$. The unique TM valence profile in LirNMC is indicative of its own characteristic chemistry. In conventional layered NMC, the capacity is almost exclusively contributed by transition metal cations and oxygen anions do not participate in the redox reaction. For these materials (at charged state), the valence of $\mathrm{Ni}$ usually is highest in the outer layer and gradually decreases as the depth increases (disregarding the very surface layer of a few nanometers, which has other chemical complexities such as the surface reconstruction effect). This is suggesting a more complete TM oxidation in the outer layer where lithium ion can be more effectively extracted. However, in oxygen-active cathode material such as LirNMC, 

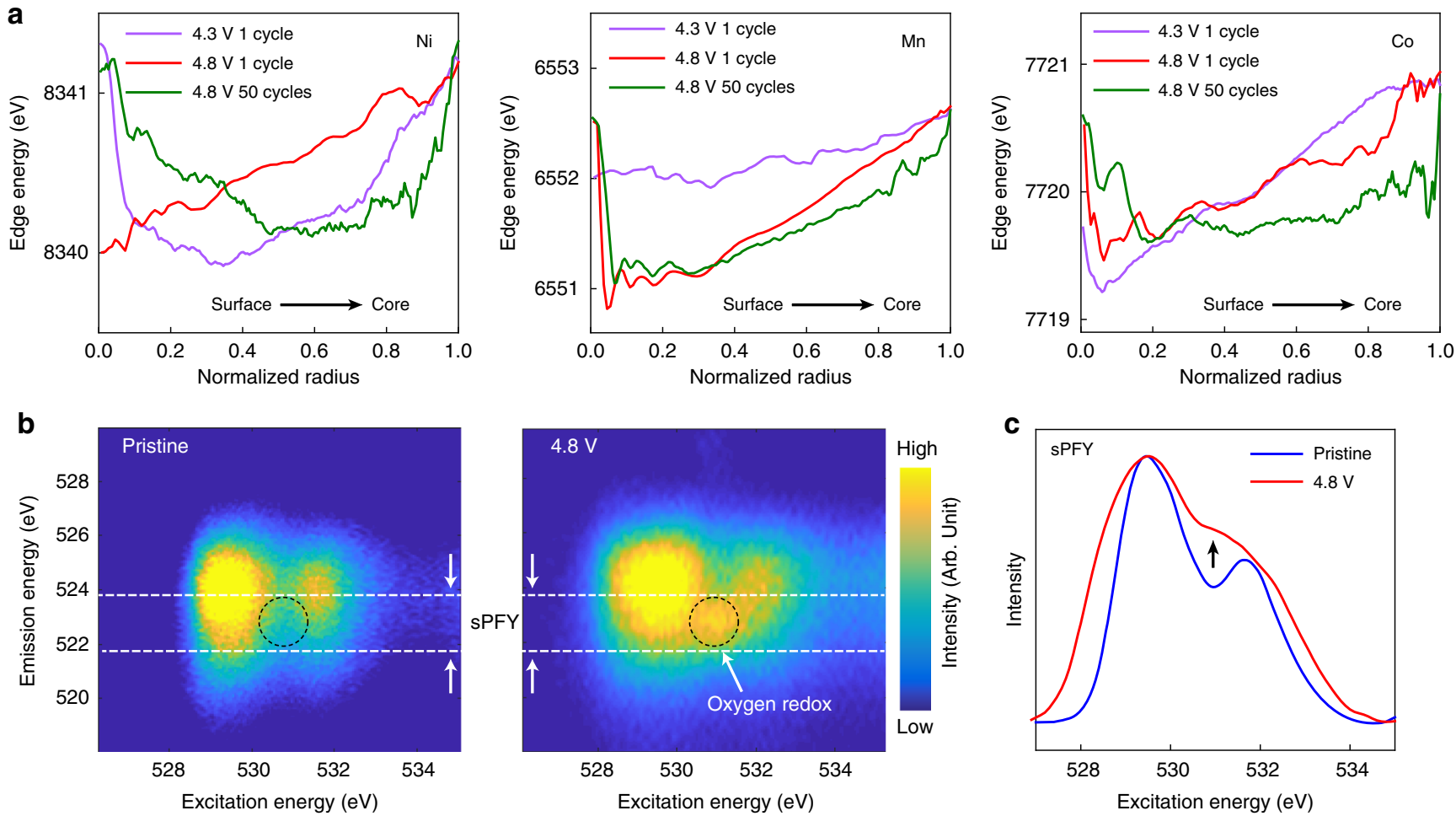

Fig. 4 Depth-dependent redox behaviors in the LirNMC layered oxide cathode. The variations of the edge energies for all the three transition metal cations ( $\mathrm{Ni}, \mathrm{Mn}$ and $\mathrm{Co}$ ) are shown in left, middle and right of panel (a), respectively. Soft x-ray RIXS data on the pristine and the charged (to $4.8 \mathrm{~V}$ in the first cycle) LirNMC samples are shown in panels (b). The comparison of the sPFY signals is shown in panel (c).

oxygen activity and the possible consequent oxygen vacancy induces an oxidation state depth profile that is significantly distinct from the conventional NMC.

Soft $x$-ray resonant inelastic $x$-ray scattering (RIXS), a powerful tool to study anion redox, is used in this study and the results are shown in Fig. $4 \mathrm{~b}$ and c. At the charged state (Fig. $4 \mathrm{~b}$ ), a peak is clearly visible at excitation energy of $531 \mathrm{eV}$ and emission energy of $523 \mathrm{eV}$, fingerprinting the formation of $\mathrm{O}^{-1}$ in the subsurface transition layer of the material upon charging to high voltage ${ }^{49-51}$. The comparison of the super partial fluorescence yield (sPFY) ${ }^{49}$ signals from the pristine and the charged samples shown in Fig. 4c clearly support this observation. Althoughoxygen activity can increase the capacity and hence the energy density of cathode material, it would eventually lead to oxygen loss, leaving behind oxygen vacancy around the transition metal cations and decreasing their valences. Such uniqueness of the LirNMC explains why the TM valence has a very different profile from that of conventional NMC despite the fact that both have nonuniform lithium distribution in the charged particle.

\section{Discussion}

To gain in-depth understanding of the voltage fade issue in LirNMC materials, synchrotron-based nano-resolution spectrotomography is used to study the material $\mathrm{Li}_{1.2} \mathrm{Ni}_{0.13} \mathrm{Mn}_{0.54^{-}}$ $\mathrm{Co}_{0.13} \mathrm{O}_{2}$ at the particle level, providing morphological, compositional, and chemical information. The unique multi-layer morphology of LirNMC is revealed in 3D with nanoscale resolution. The composition of the studied LirNMC is rather homogeneous throughout the pristine particle and not affected by charging. However, charging induces a depth-dependent profile of transition metal valence that is unique and distinguishes LirNMC from conventional NMC material. Such unique redox stratification is likely correlated with the depth-dependent association of oxygen redox with different transition metal cations.
Our results highlight the importance of particle level engineering, which could be a key to maximize the effective electrochemical activity of the active materials.

\section{Methods}

Sample preparation. $\mathrm{Li}_{1.2} \mathrm{Ni}_{0.13} \mathrm{Mn}_{0.54} \mathrm{Co}_{0.13} \mathrm{O}_{2}$ compound was prepared by a conventional solid-state reaction using stoichiometric lithium carbonate (5\% excess of lithium) and the metal hydroxide with Ni:Mn:Co of 0.13:0.54:0.13 in molar ratio. The mixture was firstly calcinated at $450{ }^{\circ} \mathrm{C}$ for $5 \mathrm{~h}$, then calcinated at $850{ }^{\circ} \mathrm{C}$ for $12 \mathrm{~h}$ in air and cooled naturally. Electrodes were prepared by spreading the slurry (N-Methyl-2-pyrrolidone as the solvent) containing active materials (80 wt.\%), acetylene carbon (10 wt.\%) and polyvinylidene difluoride (PVDF, $10 \mathrm{wt} . \%$ ) as the binder and casting it on carbon-coated aluminum foils. The electrodes were then dried overnight at $120^{\circ} \mathrm{C}$ in a vacuum oven and transferred into an Ar-filled glovebox for future use. The active mass loadings for the electrodes were $1 \mathrm{mg} \mathrm{cm}^{-2}$. CR2032 coin cells were assembled in an Ar-filled glovebox $\left(\mathrm{O}_{2}<\right.$ $0.5 \mathrm{ppm}, \mathrm{H}_{2} \mathrm{O}<0.5 \mathrm{ppm}$ ) using the composite cathode, lithium foil (MTI) anode with thickness of $250 \mu \mathrm{m}$, Celgard 2500 as the separator and electrolyte made of $1 \mathrm{M} \mathrm{LiPF}_{6}$ dissolved in ethylene carbonate (EC) and ethyl methyl carbonate (EMC) (1:2 in volume). The LirNMC electrode was charged to $4.8 \mathrm{~V}$ using a Biological potentiostat/galvanostat (SP-300). The charging rate is $\mathrm{C} / 8$ corresponding to around $37.5 \mathrm{~mA} \mathrm{~g}^{-1}$. The cell was then disassembled in the Ar-filled glovebox and the electrode was thoroughly washed by dimethyl carbonate (DMC) solvents. The washed electrode was dried overnight in the Ar atmosphere before it is further characterized.

Materials characterization. The crystal structure of the materials was characterized by powder x-ray diffraction (XPD) at 28-ID-2 beamline of the National Synchrotron Light Source II (NSLS-II) at Brookhaven National Laboratory (BNL) by a Perkin Elmer amorphous-Si flat panel detector, and the $\mathrm{x}$-ray wavelength was $0.1917 \AA$ A. XRD refinement was conducted by the Rietveld method using the TOPAS $^{52}$. The morphology of the materials was characterized by field emission scanning electron microscopy (FE-SEM, Hitachi 4800). The ex-situ and in situ xray absorption spectroscopy (XAS) experiments were performed in transmission mode using a $\mathrm{Si}$ (111) double-crystal monochromator at beamline 7-BM (QAS) of the NSLS-II at BNL. A reference spectrum for each element was simultaneously collected with the corresponding spectrum of the in-situ cells using transition metal foil. The energy calibration was carried out using the first inflection point of the Kedge spectrum of the transition metal foil as a reference. The XANES and extended 
$\mathrm{x}$-ray absorption fine-structure data were analyzed using the ATHENA software package ${ }^{53}$.

Nano-resolution spectro-tomography. X-ray micro-CT scan of a small piece of LirNMC cathode electrode was performed using Xradia Zeiss 520 Versa at Stanford Nano Shared Facilities (SNSF). In this scan, 1600 projections were collected from $-90^{\circ}$ to $+90^{\circ}$. More details of this equipment could be found elsewhere ${ }^{36}$.

The spectro-tomography was performed using the transmission $\mathrm{x}$-ray microscopy (TXM) at the beamline 6-2c of Stanford Synchrotron Radiation Lightsource (SSRL) of SLAC National Accelerator Laboratory or at beamline 18-ID (FXI) of National Synchrotron Light Source II (NSLS II) at BNL. The nominal spatial resolution of TXM is $\sim 30 \mathrm{~nm}$. The LirNMC particles were first carefully peeled off from the Al current collector and then loaded in the quartz capillary (100 microns in diameter, 10 microns in wall thickness). During the 3D spectrotomography scan, the energy scan across the absorption K-edges of Mn, Co, and $\mathrm{Ni}$ were carried out. The energy range of the incident $\mathrm{x}$-ray is $6384 \mathrm{eV}$ to $6776 \mathrm{eV}$ for $\mathrm{Mn}, 7554 \mathrm{eV}$ to $7946 \mathrm{eV}$ for $\mathrm{Co}$, and $8178 \mathrm{eV}$ to $8570 \mathrm{eV}$ for $\mathrm{Ni}$, respectively. For each of these three edges, tomography was performed at 63 different energy points. In the near edge regions, $(6534-6576 \mathrm{eV}$ for $\mathrm{Mn}, 7704-7746 \mathrm{eV}$ for $\mathrm{Co}$, and $8328-8370 \mathrm{eV}$ for $\mathrm{Ni}$, respectively), the energy step was set to $1 \mathrm{eV}$ to ensure sufficient energy resolution. The energy step size of the pre-edge and post-edge regions were set to $10 \mathrm{eV}$ for covering a relatively wide energy window for normalization of the spectra. The $3 \mathrm{D}$ tomographic reconstruction and the XANES spectra analysis were carried out using an in-house developed software package known as TXM-Wizard ${ }^{40}$

Resonant inelastic $\mathbf{x}$-ray scattering (RIXS). Soft XAS and RIXS experiments were conducted at beamline 10-1 at Stanford Synchrotron Radiation Lightsource $(\mathrm{SSRL})^{54}$. More experiment details about the soft $\mathrm{x}$-ray spectroscopic measurements can be found in our previous work ${ }^{5}$.

\section{Data availability}

The data that support the findings within this paper are available from the corresponding authors on request.

Received: 29 February 2020; Accepted: 19 November 2020;

Published online: 11 December 2020

\section{References}

1. John, B. Goodenough Facts 2019. NobelPrize.org. Nobel Media AB 2020. https://www.nobelprize.org/prizes/chemistry/2019/goodenough/facts/ (2019).

2. Lu, Z., MacNeil, D. \& Dahn, J. Layered Li[ $\left[\mathrm{Ni}_{\mathrm{x}} \mathrm{Co}_{1-2 \mathrm{x}} \mathrm{Mn}_{\mathrm{x}}\right] \mathrm{O}_{2}$ cathode materials for lithium-ion batteries. Electrochem. Solid State Lett. 4, A200-A203 (2001).

3. Thackeray, M. M. et al. $\mathrm{Li}_{2} \mathrm{MnO}_{3}$-stabilized $\mathrm{LiMO}_{2}(\mathrm{M}=\mathrm{Mn}, \mathrm{Ni}$, Co electrodes for lithium-ion batteries. J. Mater. Chem. 17, 3112-3125 (2007).

4. Assat, G. et al. Fundamental interplay between anionic/cationic redox governing the kinetics and thermodynamics of lithium-rich cathodes. Nat. Commun. 8, 2219 (2017).

5. Li, S. et al. Surface-to-bulk redox coupling through thermally driven $\mathrm{Li}$ redistribution in $\mathrm{Li}$ - and $\mathrm{Mn}$-rich layered cathode materials. J. Am. Chem. Soc. 141, 12079-12086 (2019)

6. Wang, Y., Yang, Z., Qian, Y., Gu, L. \& Zhou, H. New insights into improving rate performance of lithium-rich cathode material. Adv. Mater. 27, 3915-3920 (2015).

7. Hu, S. et al. Li-rich layered oxides and their practical challenges: recent progress and perspectives. Electrochem. Energy Rev. 2, 277-311 (2019).

8. Sathiya, M. et al. Origin of voltage decay in high-capacity layered oxide electrodes. Nat. Mater. 14, 230-238 (2015).

9. $\mathrm{Hu}, \mathrm{E}$. et al. Evolution of redox couples in Li-and Mn-rich cathode materials and mitigation of voltage fade by reducing oxygen release. Nat. Energy 3 , 690-698 (2018).

10. Singer, A. et al. Nucleation of dislocations and their dynamics in layered oxide cathode materials during battery charging. Nat. Energy 3, 641-647 (2018).

11. Hong, J. et al. Metal-oxygen decoordination stabilizes anion redox in Li-rich oxides. Nat. Mater. 18, 256-265 (2019).

12. Wei, C. et al. Mesoscale battery science: the behavior of electrode particles caught on a multispectral X-ray camera. Acc. Chem. Res. 51, 2484-2492 (2018).

13. Yang, F. et al. Nanoscale morphological and chemical changes of high voltage lithium-manganese rich NMC composite cathodes with cycling. Nano Lett. 14, 4334-4341 (2014).

14. Tian, C. et al. Depth-dependent redox behavior of $\mathrm{LiNi}_{0 .}{ }_{6} \mathrm{Mn}_{0.2} \mathrm{Co}_{0.2} \mathrm{O}_{2}$. J. Electrochem. Soc. 165, A696-A704 (2018).
15. Tian, C., Lin, F. \& Doeff, M. M. Electrochemical characteristics of layered transition metal oxide cathode materials for lithium ion batteries: surface, bulk behavior, and thermal properties. Acc. Chem. Res. 51, 89-96 (2017).

16. Wei, C. et al. Thermally driven mesoscale chemomechanical interplay in $\mathrm{Li}_{0.5} \mathrm{Ni}_{0.6} \mathrm{Mn}_{0.2} \mathrm{Co}_{0.2} \mathrm{O}_{2}$ cathode materials. J. Mater. Chem. 6, 23055-23061 (2018).

17. Alvarado, J. et al. Thermal stress-induced charge and structure heterogeneity in emerging cathode materials. Mater. Today 35, 87-98 (2019).

18. Besli, M. M. et al. Mesoscale chemomechanical interplay of the $\mathrm{LiNi}_{0.8} \mathrm{Co}_{0.15} \mathrm{Al}_{0.05} \mathrm{O}_{2}$ cathode in solid-state polymer batteries. Chem. Mater. 31, 491-501 (2018).

19. Johnson, C. S. Charging Up lithium-ion battery cathodes. Joule 2, 373-375 (2018).

20. Mu, L. et al. Propagation topography of redox phase transformations in heterogeneous layered oxide cathode materials. Nat. Commun. 9, 2810 (2018).

21. Lin, F. et al. Metal segregation in hierarchically structured cathode materials for high-energy lithium batteries. Nat. Energy 1, 15004 (2016).

22. $\mathrm{Xu}, \mathrm{Y}$. et al. In situ visualization of state-of-charge heterogeneity within a $\mathrm{LiCoO}_{2}$ particle that evolves upon cycling at different rates. ACS Energy Lett. 2, 1240-1245 (2017).

23. Yang, Y. et al. Quantification of heterogeneous degradation in Li-Ion batteries. Adv. Energy Mater. 9, https://doi.org/10.1002/aenm.201900674 (2019).

24. Xia, S. et al. Chemomechanical interplay of layered cathode materials undergoing fast charging in lithium batteries. Nano Energy 53, 753-762 (2018).

25. Wang, J. et al. Visualization of anisotropic-isotropic phase transformation dynamics in battery electrode particles. Nat. Commun. 7, 12372 (2016).

26. Weker, J. N. et al. In situ nanotomography and operando transmission X-ray microscopy of micron-sized Ge particles. Energy Environ. Sci. 7, 2771-2777 (2014).

27. $\mathrm{Xu}, \mathrm{Z}$. et al. Charge distribution guided by grain crystallographic orientations in polycrystalline battery materials. Nat. Commun. 11, 83 (2020).

28. Tsai, P.-C. et al. Single-particle measurements of electrochemical kinetics in NMC and NCA cathodes for Li-ion batteries. Energy Environ. Sci. 11, 860-871 (2018).

29. Yu, Y.-S. et al. Three-dimensional localization of nanoscale battery reactions using soft X-ray tomography. Nat. Commun. 9, 1-7 (2018).

30. House, R. A. et al. Superstructure control of first-cycle voltage hysteresis in oxygen-redox cathodes. Nature 577, 502-508 (2020).

31. Finegan, D. P. et al. Spatial quantification of dynamic inter and intra particle crystallographic heterogeneities within lithium ion electrodes. Nat. Commun. 11, 631 (2020).

32. Xu, R. et al. Heterogeneous damage in Li-ion batteries: experimental analysis and theoretical modeling. J. Mech. Phy. Solids 129, 160-183 (2019).

33. Mao, Y. et al. High-voltage charging-induced strain, heterogeneity, and microcracks in secondary particles of a nickel-rich layered cathode material. Adv. Funct. Mater. 29, 1900247 (2019).

34. Xu, Y. et al. Structural integrity-searching the key factor to suppress the voltage fade of Li-rich layered cathode materials through 3D X-ray imaging and spectroscopy techniques. Nano Energy 28, 164-171 (2016).

35. Chen, C.-J. et al. The origin of capacity fade in the $\mathrm{Li}_{2} \mathrm{MnO}_{3} \cdot \mathrm{LiMO}_{2}(\mathrm{M}=\mathrm{Li}$, $\mathrm{Ni}, \mathrm{Co}, \mathrm{Mn}$ ) microsphere positive electrode: an operando neutron diffraction and transmission X-ray microscopy study. J. Am. Chem. Soc. 138, 8824-8833 (2016).

36. Semnani, S. J. \& Borja, R. I. Quantifying the heterogeneity of shale through statistical combination of imaging across scales. Acta Geotech. 12, 1193-1205 (2017).

37. Yan, P. et al. Atomic-resolution visualization of distinctive chemical mixing behavior of $\mathrm{Ni}, \mathrm{Co}$, and $\mathrm{Mn}$ with $\mathrm{Li}$ in layered lithium transition-metal oxide cathode materials. Chem. Mater. 27, 5393-5401 (2015).

38. $\mathrm{Yu}, \mathrm{H}$. et al. Direct atomic-resolution observation of two phases in the $\mathrm{Li}_{1}$ ${ }_{2} \mathrm{Mn}_{0 .}{ }_{56} \mathrm{Ni}_{0 .}{ }_{166} \mathrm{Co}_{0.067} \mathrm{O}_{2}$ cathode material for lithium-ion batteries. Angew. Chem. Int. Ed. 52, 5969-5973 (2013).

39. Meirer, F. et al. Three-dimensional imaging of chemical phase transformations at the nanoscale with full-field transmission X-ray microscopy. J. Synchrotron Radiat. 18, 773-781 (2011).

40. Liu, Y. et al. TXM-Wizard: a program for advanced data collection and evaluation in full-field transmission X-ray microscopy. J. Synchrotron Radiat. 19, 281-287 (2012).

41. Nelson, G. J. et al. Three-dimensional mapping of nickel oxidation states using full field x-ray absorption near edge structure nanotomography. Appl. Phys. Lett. 98, 173109 (2011).

42. Gent, W. E. et al. Persistent state-of-charge heterogeneity in relaxed, partially charged $\mathrm{Li}_{1-\mathrm{x}} \mathrm{Ni}_{1 / 3} \mathrm{Co}_{1 / 3} \mathrm{Mn}_{1 / 3} \mathrm{O}_{2}$ secondary particles. Adv. Mater. 28, 6631-6638 (2016).

43. Koettgen, J., Bartel, C. \& Ceder, G. Computational investigation of chalcogenide spinel conductors for all-solid-state Mg batteries. Chem. Commun. 56, 1952-1955 (2020). 
44. Kao, T. L. et al. Nanoscale elemental sensitivity study of $\mathrm{Nd}_{2} \mathrm{Fe}_{14} \mathrm{~B}$ using absorption correlation tomography. Microsc. Res. Tech. 76, 1112-1117 (2013).

45. Zhao, S., Yan, K., Zhang, J., Sun, B. \& Wang, G. Reviving reaction mechanism of layered lithium-rich cathode materials for high-energy lithium-ion battery. Angew. Chem. Int. Ed. 10, 1002 (2020).

46. Gent, W. E. et al. Coupling between oxygen redox and cation migration explains unusual electrochemistry in lithium-rich layered oxides. Nat. Commun. 8, 1-12 (2017).

47. Seo, D.-H. et al. The structural and chemical origin of the oxygen redox activity in layered and cation-disordered Li-excess cathode materials. Nat Chem. 8, 692 (2016).

48. Bréger, J. et al. Short-and long-range order in the positive electrode material, $\mathrm{Li}(\mathrm{NiMn})_{0.5} \mathrm{O}_{2}$ : a joint $\mathrm{X}$-ray and neutron diffraction, pair distribution function analysis and NMR study. J. Am. Chem. Soc. 127, 7529-7537 (2005).

49. Dai, K. et al. High reversibility of lattice oxygen redox quantified by direct bulk probes of both anionic and cationic redox reactions. Joule 3, 518-541 (2019).

50. Qiao, R. et al. High-efficiency in situ resonant inelastic x-ray scattering (iRIXS) endstation at the advanced light source. Rev. Sci. Instrum. 88, 033106 (2017).

51. Wu, J. et al. Fingerprint oxygen redox reactions in batteries through highefficiency mapping of resonant inelastic X-ray scattering. Condens. Matter 4, 5 (2019).

52. Coelho, A. A. TOPAS and TOPAS-Academic: an optimization program integrating computer algebra and crystallographic objects written in C. J. Appl. Crystallogr. 51, 210-218 (2018).

53. Ravel, B. \& Newville, M. A. T. H. E. N. A. ATHENA, ARTEMIS, HEPHAESTUS: data analysis for X-ray absorption spectroscopy using IFEFFIT. J. Synchrotron Radiat. 12, 537-541 (2005).

54. Lee, S.-J. et al. Soft X-ray spectroscopy with transition-edge sensors at Stanford Synchrotron Radiation Lightsource beamline 10-1. Rev. Sci. Instrum. 90, 113101 (2019).

\section{Acknowledgements}

Use of the Stanford Synchrotron Radiation Lightsource, SLAC National Accelerator Laboratory, is supported by the U.S. Department of Energy, Office of Science, Office of Basic Energy Sciences under Contract No. DE-AC02-76SF00515. The work at Brookhaven National Laboratory is supported by the Assistant Secretary for Energy Efficiency and Renewable Energy, Vehicle Technology Office of the U.S. DOE through the Advanced Battery Materials Research (BMR) Program under contract No. DESC0012704. J. Zhang, K. Zhang, and Q. Yuan are partly supported by the National Key Research and Development Program of China Grant No. 2016YFA0400900. Part of this work was performed at the Stanford Nano Shared Facilities, supported by National Science Foundation Grant ECCS-1542152. The engineering support from D. Van Campen, D. Day and V. Borzenets for the TXM experiment at beamline 6-2C of SSRL is gratefully acknowledged. This research used resources 7-BM (QAS), 18-ID (FXI), and 28ID-2 (XPD) of the National Synchrotron Light Source II, a U.S. Department of Energy (DOE) Office of Science User Facility operated for the DOE Office of Science by
Brookhaven National Laboratory under Contract No. DE-SC0012704. C.J. Titus is supported by the U.S. Department of Energy Office of Basic Energy Sciences proposal No. 100487.

\section{Author contributions}

Y.L., E.H., and X.-Q.Y. supervised this study. Q.W., X.W., S.T., E.H. performed electrochemical tests, and sample preparation. J.Z., S.L., Z.J., K.Z., Q.Y., X.X., and Y.L. performed the spectro-tomographic measurements and analysis. S.L., S.-J.L., D.N., and J.-S.L. conducted the soft XAS and RIXS measurements. C.J.T. and K.D.I. contributed to the TES development for RIXS measurements. X.Y., P.P. and X.-Q.Y. contributed to the scientific interpretation of the synchrotron data. J.Z., Q.W., E.H., and Y.L. wrote the manuscript with assistance from coauthors. J.Z. and Q.W. contributed equally to this work

\section{Competing interests}

The authors declare no competing interests.

\section{Additional information}

Supplementary information is available for this paper at https://doi.org/10.1038/s41467 020-20198-w.

Correspondence and requests for materials should be addressed to Q.Y., E.H. or Y.L.

Peer review information Nature Communications thanks the anonymous reviewers for their contribution to the peer review of this work.

Reprints and permission information is available at http://www.nature.com/reprints

Publisher's note Springer Nature remains neutral with regard to jurisdictional claims in published maps and institutional affiliations.

(c) (i)

Open Access This article is licensed under a Creative Commons Attribution 4.0 International License, which permits use, sharing, adaptation, distribution and reproduction in any medium or format, as long as you give appropriate credit to the original author(s) and the source, provide a link to the Creative Commons license, and indicate if changes were made. The images or other third party material in this article are included in the article's Creative Commons license, unless indicated otherwise in a credit line to the material. If material is not included in the article's Creative Commons license and your intended use is not permitted by statutory regulation or exceeds the permitted use, you will need to obtain permission directly from the copyright holder. To view a copy of this license, visit http://creativecommons.org/ licenses/by/4.0/.

(C) The Author(s) 2020 